\title{
Asbestos, Simian virus 40 and malignant mesothelioma
}

\section{S C Stenton}

Department of Respiratory Medicine and Regional Unit for Occupational Lung Disease, Royal Victoria Infirmary and University of Newcastle upon Tyne, Newcastle upon Tyne, UK

Introductory article

Simian virus 40 large T antigen (SV40LTAg) primer specific DNA amplification in human pleural mesothelioma tissue

\section{Pepper, B Jasani, H Navabi, D Wynford-Thomas, AR Gibbs}

Background. DNA sequences and immunoreactivity associated with Simian virus 40 transforming factors, large T and small t antigens (SV4OLTAg), suggestive of an aetiopathogenetic link have been identified in fresh frozen tissue of a high proportion of recent cases of pleural mesotheliomas from the United States, Italy and Germany. SV40 is not normally infective in man though it can transform human cells in tissue culture. A large cohort of people in the western world was accidentally parenterally inoculated with live SV40 through contaminated polio vaccines given between 1959 and 1961, and this might be a factor in the current continuing rise in the incidence of mesothelioma in the United States, Britain and Europe. The present study investigated the presence of SV40LTAg DNA in recently diagnosed cases of mesothelioma in Britain and the feasibility of detecting the SV40 DNA in archival tissue for retrospective analysis of cases in the peri-vaccination period. Methods. DNA was extracted from fresh frozen and/or rehydrated formalin fixed, paraffin embedded tissue sectionsfrom nine recently diagnosed cases of mesothelioma, nine cases of pulmonary adenocarcinoma, and three reactive pleurae, and amplified by the polymerase chain reaction (PCR) using the primer pairs used previously on fresh frozen tissues - namely, the SV primer set directed at the LTAg gene sequence unique to SV40 and the PYV primer set directed at a sequence shared by SV40 and papovavirus strains BK and JC, respectively. Results. PCR positivity with the SV primer set was restricted to four of the nine cases of mesothelioma. In contrast, six of the nine mesotheliomas, two of the nine adenocarcinomas, and one of the three reactive pleurae showed positivity with the PYV primers. The fresh frozen and corresponding formalin fixed, paraffin embedded tissue results concorded well with each other. Conclusions. Our data provide evidence for the association of SV40LTAg primer specific DNA with human pulmonary mesothelioma in the British population. (Thorax 1996;51:1074-6)

A bout 1000 cases of malignant mesothelioma are currently reported each year in Britain but the incidence of the disease continues to rise and is anticipated to peak at up to 3000 cases per year round the year 2020 (fig 1). ${ }^{1}$ This reflects the extensive use of amphibole asbestos (crocidolite and amosite) up to the 1970s and the long latency of the tumour which is typically 30 years or more from the time of first exposure. The association between work with amphibole asbestos and the later development of a malignant mesothelioma is one of the closest in cancer epidemiology. Its incidence amongst non-exposed populations is of the order of one per million per year, but lifetime risks of up to $10 \%$ have been reported in groups of heavily exposed workers. ${ }^{2}$ The tumour is not associated with cigarette smok ing and, apart from the subject of the introductory article, no other cofactors have been reported. It has become customary to cite asbestos as a "complete carcinogen" for the mesothelium, and to contrast this with its effect on the airway epithelium where there is still considerable debate about its independent role in the causation of cancer and where cigarette smoking is an important cofactor ${ }^{3}$

With this background it comes as something of a surprise to be presented with the data in the introductory article which suggest that there are factors other than asbestos which might be of considerable aetiological importance for the development of malignant mesothelioma. ${ }^{4} \mathrm{~T}$ he hypothesis is readily plausible because our current knowledge of the pathogenesis of mesothelioma does not encompass any satisfactory explanation for (1) its dose-response relationship with 


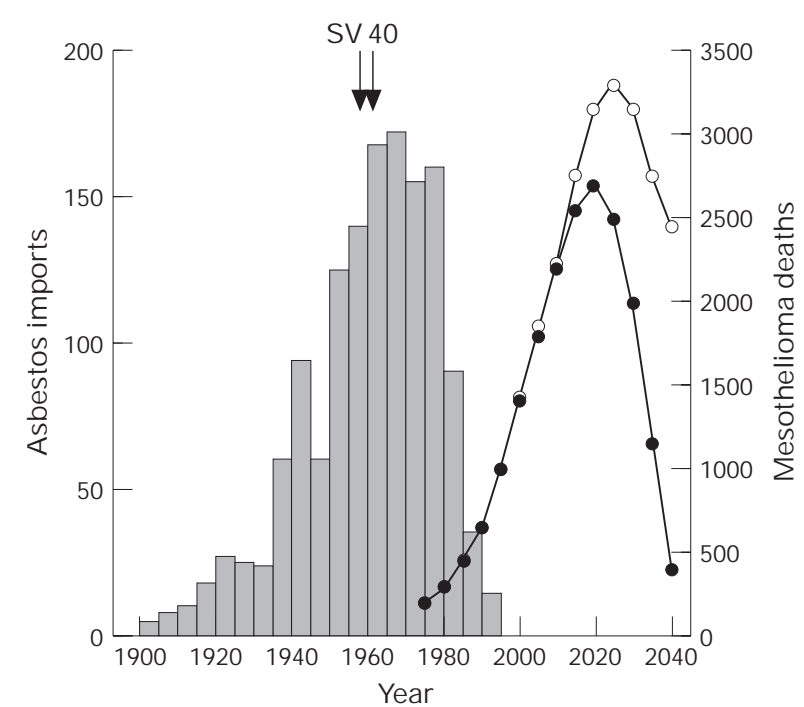

Figure 1 UK asbestos imports (histogram) and predicted mesothelioma deaths for men born before $1953(\boldsymbol{O})$ and for
all men $(\bigcirc)$. Adapted with permission from Peto et al. ${ }^{1}$

tumours often developing after relatively low exposures, (2) its long latency, and (3) its occasional occurrence in the absence of any apparent mineral fibre exposure. F urthermore, oncologists now regard the development of malignancy as a multi-step process, and the very long latent period of mesotheliomas suggests that there are multiple pathogenetic steps, at any one of which pomultiple pathogenetic steps, at any one of
tentiation or other interaction might occur.

\section{SV40 and human cancer}

The introductory article 4 has added to a growing body of knowledge suggesting that Simian virus 40 (SV40) D N A can be identified in several types of human malignancy. ${ }^{6-12}$ The techniques used in the study by Pepper nancy. ${ }^{6-12}$ The techniques used in the study by Pepper
and colleagues ${ }^{4}$ were chosen to replicate previous work,
and although they are not described in detail in their rapid communication, they can be largely inferred. The region of the viral genome under investigation codes for its large T antigen (TAg; fig 2). It is well characterised and appropriate primer seque reactions ( $P C R$ ) are available $P C R$ involves adding oligonucleotide "primer" sequences from two areas of a gene to the material under investigation together with a DNA polymerase. The region of the gene between the two primer sequences is amplified, provided it is present in the parent sample. In this study the primers were expected to amplify a 105 base-pair sequence and the reaction produced D N A fragments of approximately the correct length in four of nine mesotheliomas.

SV 40 is a small double stranded DNA virus which causes asymptomatic infection in the kidneys of rhesus monkeys. It belongs to the group of papovaviruses which include mouse polyomavirus, human BK and JC viruses, and papillomaviruses. BK and JC viruses (named after the subjects in whom they were identified) commonly cause asymptomatic human infection with $70-80 \%$ of adults being seropositive. ${ }^{13}$ They are structurally very similar to SV 40 with $69 \%$ homology of their DNA BK causes cystitis and nephritis in immunosuppressed BK causes cystitis and nephritis in immunosuppressed patients and JC is associated with progressive multifocal leucoencephalopathy. These papovaviruses share with adenoviruses (another DNA virus of vertebrates) a potent ability to induce tumours their natural hosts. Papillomaviruses are associated with

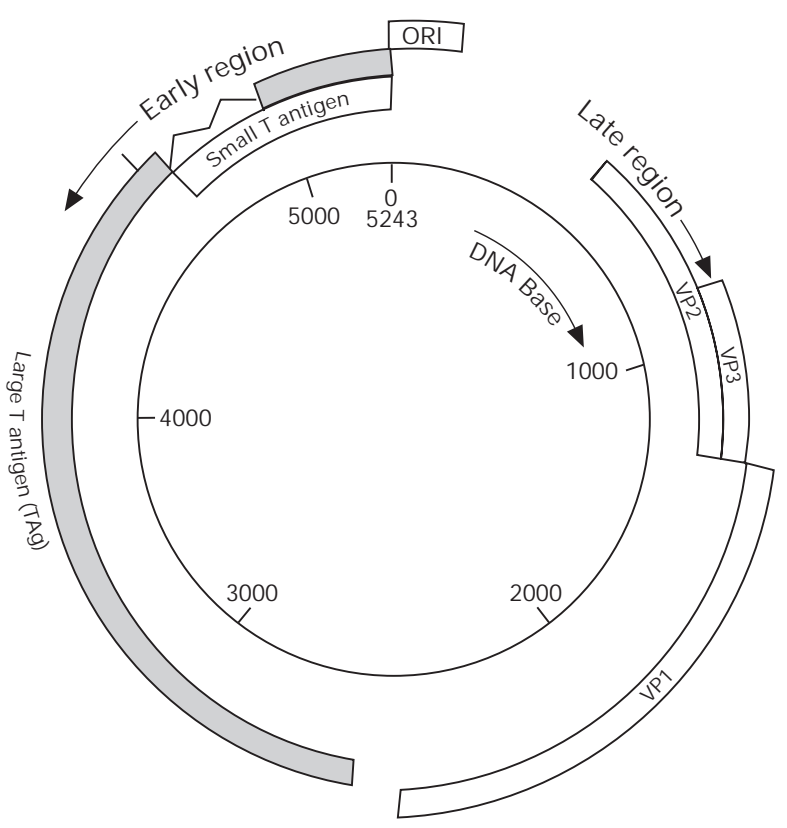

Figure 2 Structure of SV40 genome. Viral replication begins at ORI (the origin) and proceeds in both directions. The early antigens code anticlockwise and the late protein coat antigens code clockwise. Adapted with permission

cervical cancer but there is, to date, no convincing evidence that other papovaviruses cause malignancy in humans.

SV 40-like DN A was identified in human tumours in SV 40 -like DNA was identified in human tumours in
1992 by Bergsagel and colleagues who were looking for JC- or BK-like DNA in childhood choroid plexus tumours and ependymomas. ${ }^{6}$ When PCR was performed with polyomavirus (PVY) primers under conditions of low stringency - that is, allowing DNA amplification despite some base-pair mismatching amplification products of approximately the correct size were detected. H owever, when a portion of the amplified DNA was sequenced it was found to be identical to that of a region of SV $40 \mathrm{TAg}$ and different from the DNA of the JC and BK viruses in several respects. When the authors repeated the PCR under conditions of high stringency using SV 40 primers they detected DNA which hybridised with a further SV40 oligonucleotide sequence in 20 of 31 of the tumours. Immunohistochemical staining revealed nuclear SV 40 TAg in seven of 11 cases. Seventeen tumour samples were restudied blindly in a different laboratory using primers for three different parts of the SV 40 genome and viral DNA was found in 14, including all eight which had previously been considered positive The and, in one case, produced what were regarded as typical ral cytopathic effects.

At around the same time SV 40 was noted to induce mesotheliomas when inoculated into the pleural cavities of hamsters $\mathrm{s}^{14}$ and so $\mathrm{C}$ arbone and coworkers extended Bergsagel's observations with a study of human mesotheliomas. ${ }^{11}$ SV 40-like D N A was identified in 29 of 48 tumours but in only one of 28 lung tissue samples from the same subjects, and in none of 48 other solid tumours. A 132 base-pair DNA segment which was sequenced showed only a single nucleotide difference from SV 40 TAg DNA. Nuclear TAg was detected immunohistochemically in 11 of 14 tumours, and all of 26 serum samples contained anti-TAg antibodies. One further study identified SV 40-like D N A in eight of 11 malignant mesotheliomas and in none of seven control samples. ${ }^{12}$ 


\begin{tabular}{lccc}
\hline Table 1 Frequency of identification of SV40-like DNA \\
\hline Tissue type & SV40-like DNA & Reference \\
\hline Mesothelioma & $29 / 48$ & $(60 \%)$ & 11 \\
& $4 / 9$ & $(37 \%)$ & 4 \\
& $8 / 11$ & $(73 \%)$ & 12 \\
Choroid plexus/ependymoma & $0 / 50$ & $(0 \%)$ & 15 \\
Ependymoma & $33 / 47$ & $(70 \%)$ & 6,9 \\
Osteosarcoma & $0 / 10$ & $(0 \%)$ & 16 \\
Lung cancer/adenocarcinoma & $40 / 126$ & $(32 \%)$ & 8 \\
Normal brain & $0 / 39$ & $(0 \%)$ & 4,11 \\
Normal lung & $1 / 20$ & $(5 \%)$ & 6,9 \\
Peripheral blood & $1 / 28$ & $(4 \%)$ & 11 \\
\hline
\end{tabular}

On the other hand, Strickler and colleagues failed to replicate these findings. ${ }^{15} \mathrm{~T}$ hey performed PC R on tissue from 50 mesotheliomas using the same primers as Bergsagel and others, and failed to identify any amplified D N A capable of hybridising with SV 40 sequences. Their results could not be explained by any obvious artefact; 48 of the 50 specimens amplified DNA using primers for B-globin genes, indicating that amplifiable DNA for B-globin genes, indicating that amplifiable DNA
was present, and positive results were obtained from was present, and positive results were obtained from
Simian and human cell lines known to have SV 40 D N A incorporated into their genome. Serum SV 40 antibodies assayed using a viral culture plaque inhibition assay were detected in only three of 34 samples. Other workers have failed to identify SV 40 DNA in brain tumours (table 1$)^{16}$ and the contradictory observations need to (table 1$)^{16}$ and

Amplifying DNA sequences from tissue samples which are often fixed in formaldehyde and embedded in paraffin is not a simple matter and the potential exists for misidentification and for false positive and false negative results. The introductory article showed only that the SV 40 primers amplified DNA sequences of approximately the correct length and did not demonstrate that they contained SV 40-like sequences. H owever, the DNA sequence has been confirmed in other ever, the DN A sequence has been confirmed in other
laboratories and it seems unlikely that the PCR primers laboratories and it seems unlikely that the PCR primers
are amplifying either JC or BK viruses or parts of the normal human genome. However, and despite the structural similarities with SV 40, it is possible that the DNA originates from a previously unrecognised human contamination by laboratory SV 40 strains is also possible as PC R is exquisitely sensitive to small amounts of extraneous DNA, and SV40 is a commonly used laboratory virus. The SV 40-like D N A from some bone tumours was found to have an extra copy of a 72 basepair enhancer region which is found in laboratory strains but not in wild SV $40 .{ }^{8} \mathrm{O}$ the other hand, sequences identified in brain tumours matched more than one strain of wild virus and were not homologous with laboratory strains, and were thus not due to contamination. Laboratory contamination also does not readily explain the tissue specificity of the findings.

The amplification of extracted SV40 DNA appears to be highly reproducible between laboratories with discordant results having been reported in only $3 \%$ of samples, but the method of extraction of DNA from tissue samples does affect the identification rate and might explain interlaboratory differences. ${ }^{8}$ T he laboratories which identified viral DNA in mesothelial tissue also found immunochemical evidence of viral protein production whereas other laboratories found neither raising the possibility of geographical differences in viral prevalence. However, the serological and immunohistochemical tests are not well characterised and the
$100 \%$ identification of antibodies in one laboratory and the almost $0 \%$ identification in another suggests that the differences are technical.

D oubt also remains about the extent of tissue infection with viral DNA. The mesothelioma data suggest that it is localised to the tissue of origin of the tumour, ${ }^{11}$ whereas the presence of viral D N A in blood from patients with osteosarcomas suggests that it is more widely disosteosarcomas suggests that it is more widely dis-
tributed. ${ }^{8}$ It is not known whether the entire viral genome is usually present, though that sometimes seems to be the case, ${ }^{7}$ or whether it is truly incorporated into the host genome. Incorporation of SV 40 into host D N A occurs commonly in vitro but in human bone tumours the DNA appeared to be present in short sequences and might have been episomal ${ }^{8}$ At this stage it seems clear that the observations of SV 40 DNA in human tumours can neither be accepted without reservation nor dismissed, and that further work is necessary to refine and standardise the identification techniques and to explain the discrepancies. In the meantime, it is worth considering how Simian viral DNA might have come to be present in tumours and whether there are clues suggesting that it might have an aetiologically significant role.

\section{Human exposure to SV40 and oncogenic}

properties

SV 40 was identified in 1961 in the kidney cells of rhesus monkeys which were used from the early 1950s to culture poliovirus for the newly developed Salk parenteral vaccine. ${ }^{1718} \mathrm{SV} 40$ produced no cytopathic effects in the Simian kidney cells and by the time its presence was recognised $62 \%$ of the USA population had been immunised with potentially infected vaccines. ${ }^{18}$ The extent of contamination of vaccines administered before 1961 is not known but, as $60 \%$ of rhesus monkeys used to culture poliovirus were infected and as SV 40 is more resistant than poliovirus to chemical inactivation, it is thought that up to $30 \%$ contained live SV $40 .{ }^{18} \mathrm{H}$ igh levels of infective viral particles (10000 per ml) were found in some samples, and SV40 antibodies were found in $20 \%$ of vaccinated schoolchildren. ${ }^{19}$ Oral polio vaccines were not licensed for clinical use until 1962 when SV 40 had been largely eliminated from the culture system, and were probably not contaminated to any significant extent. Contamination of some adenovirus and hepatitis vaccines has been reported. ${ }^{817}$

$M$ ost patients currently suffering from mesotheliomas are old enough to have received contaminated polio vaccines but that is not the case for the children with cerebral tumours in whom viral DNA was identified, and this suggests that there are other potential sources of infection. Support for this comes from the identification of SV 40 antibodies in $2 \%$ of blood samples obtained before the introduction of polio vaccines, in $3 \%$ of schoolchildren born after vaccines were cleared of SV 40 , and in $4 \%$ of elderly patients with no history of polio vaccination..$^{19} \mathrm{~T}$ he significance of these serological studies is not entirely certain because of the possibility of cross reactions with anti-JC and anti-BK antibodies. Contact with monkeys can lead to infection but the range of natural SV40 host animals is narrow and seropositivity rate. The virus can replicate in the nose seropositivity rate. The virus can replicate in the nose
and intestinal tracts of humans and can be shed for and intestinal tracts of humans and can be shed for
several weeks after infection, but nothing is known about whether transmission between humans occurs. ${ }^{18}$

Shortly after its identification, SV 40 was found to be highly oncogenic when injected into immature ham- 
sters. ${ }^{20}$ This observation caused considerable concern and several studies of vaccinated cohorts were established. Most showed no greater prevalence of tumours amongst immunised subjects, though one study did report an increased incidence of neural tumours amongst the offspring of mothers vaccinated during amongst ${ }^{21}$ A more recent compa of the cohor of infants most likely to have received contaminated polio vaccines with the next closest five year age group is reported to have confirmed the absence of any increased incidence of tumours including mesotheliomas, ${ }^{22}$ but the power of all these studies to detect an increased risk of uncommon tumours is limited. Sporadic cases of SV40 immunoreactivity in human have been reported ${ }^{23}$ but, until recently, the almost universally held view was that SV 40 does not have a role in human oncogenesis.

In hamsters SV 40 induces a limited range of sarcomas but does not induce carcinomas. Intracerebral injection gives rise to ependymomas ${ }^{24}$ and SV 40 transgenic mice produce progeny with a very high incidence of choroid plexus tumours. ${ }^{25}$ Intra genic sarcomas and other tumours ${ }^{20}$ Intrapleural SV 40 produced mesotheliomas in all 12 injected hamsters in one study, ${ }^{14}$ suggesting that SV 40 is, if anything, a more potent cause of mesotheliomas in the hamster than asbestos. It is noteworthy that the tumours which are most readily induced in hamsters are also those from which viral DN A has been isolated in humans. It might also be relevant that the mesothelium and ren. cells (the Simian host tissue of the virus) are unusual in being mesodermally derived tissues with epithelial characteristics. This might suggest either a viral trophism for these particular tissues or the expression of gene products with which the virus might interact to produce malignant changes.

In vitro studies also support the possibility of a role for SV40 in inducing malignant change. M ost cells cultured in vitro grow as a monolayer on culture plates for only a limited number of divisions before the cells for only a limited number of divisions before the cells become senescent. Some cells infected with viruses
undergo "transformation" and display loss of contact undergo "transformation" and display loss of contact inhibition and anchorage dependence, reduced requirements for serum growth factors, and the ability to replicate indefinitely (immortality). Some transformed cells can also induce tumours when injected into animals. SV40 genes have been used to transform or immortalise more than 20 different tissue types, including mesothelial cells. ${ }^{26}$ T he immortalised cells displayed multiple chromosomal abnormalities but retained mesothelial characteristics such as keratin and vimentin expression and growth inhibition by asbestos fibres. They did not produce tumours when injected into mice and could not be induced to become tumorigenic by exposure to asbestos. ${ }^{27}$ They did, however, become malignant when co-transfected with a ras oncogene, a cytoplasmic G T P-ase with growth stimulating properties. ${ }^{27} \mathrm{~T}$ his suggests that expression of SV 40 genes is able to take mesothelial cells one step along a pathway towards full malignant expression but that other steps are often necessary.

\section{Structure of SV40 and mechanism of}

\section{oncogenesis}

Viruses such as SV 40 carry only a limited amount of genetic material and depend heavily on host cell functions for their replication. T his requires them to subvert normal cellular mechanisms towards virus replication, and each virus has developed proteins which serve this function. They have structural similarities and are termed "early" antigens because of the timing of their expression during the infective cycle. They are distinguished from "late" antigens which code for viral coat proteins. In the case of SV40, the large tumour antigen (large $\mathrm{T}$ antigen or TAg) is the principal early (A) is the principal early protein. It is multifunctional and in "permissive" cells - that is, those which allow viral replication - it binds to viral DNA and in the presence of other cellular proteins unwinds it and allows replication. ${ }^{28} \mathrm{TAg}$ also binds and inactivates a number of tumour suppressor gene products which normally have inhibitory influences on the enzymes necessary for DNA replication, it upregulates the expression of insulin growth factor i stimulates cell division, and has sel stimulates cell division, and erties. A small $t$ antigen is produced by differential splicing of RN A of the same gene and has an important role in facilitating TAg effects.

The tumorigenic properties of D N A viruses represent the outcome of their replication strategy in cells which, for unknown reasons, do not allow viral replication. Under these circumstances the viral DNA can become randomly incorporated into the host genome an nome and, if its products are expressed, they can inactivate suppressor gene products and so release a stimulus to unregulated cell growth. ${ }^{29} \mathrm{H}$ uman cells are semi-permissive for SV 40 and allow both viral replication and D N A incorporation into the host genome.

Several nuclear proteins are known to be bound and inactivated by viral early antigens such as SV 40 large T antigen. The best characterised of these are p110, the protein product of the retinoblastoma gene, and p53. The retinoblastoma gene sited on the long arm of chromosome 13 is the prototypic tumour suppressor gene and is associated with retinoblastomas, osteosarcomas, and various other tumours, though not mesotheliomas..$^{30}$ It is under-phosphorylated in the early phases of cell division and becomes progressively more phosphorylated as di phosphorylated as division progresses. It is thought that a reduction in the levels of hypophosphorylated p110
by SV40 TAg binding neutralises its growth arresting properties. The amino acid residues 105-115 which are required for SV 40 binding to $p 110$ are also essential for its cell transforming properties. They are homologous with sequences on the adenovirus early region $1 \mathrm{~A}$ protein which are essential for that virus's transforming properties and binding of p110, suggesting a common mechanism for viral cell transformation (fig 3) ${ }^{29}$ The sequences of SV40 DNA which have been amplified from human tumours code for the p110 binding region. P53 is the most important tumour suppressor gene involved in human cancer with more than $50 \%$ of tumours exhibiting loss of function. ${ }^{31}$ Its levels are increased by breaks in DNA strands and this leads either to arrest of cell replication for a suffcient period to allow DN A to be repaired, or to cell death (apoptosis). P53 has thus been named the "guardian of the genome". ${ }^{5}$ SV 40 and other viral proteins bind to p53 and inactivate it, reducing its DNA repair, apoptotic, and growth inhibitory functions, and might in this way contribute to the development of malignancy.

\section{Pathogenesis of malignant mesothelioma}

M odern concepts of oncogenesis emphasise its multistep nature. ${ }^{5} \mathrm{An}$ abnormality of the genome is an important initiating event, but this does not lead to tumour development unless the altered cell escapes from normal growth control mechanisms before either dying or undergoing DN A repair. A wide variety of proto-onco- 


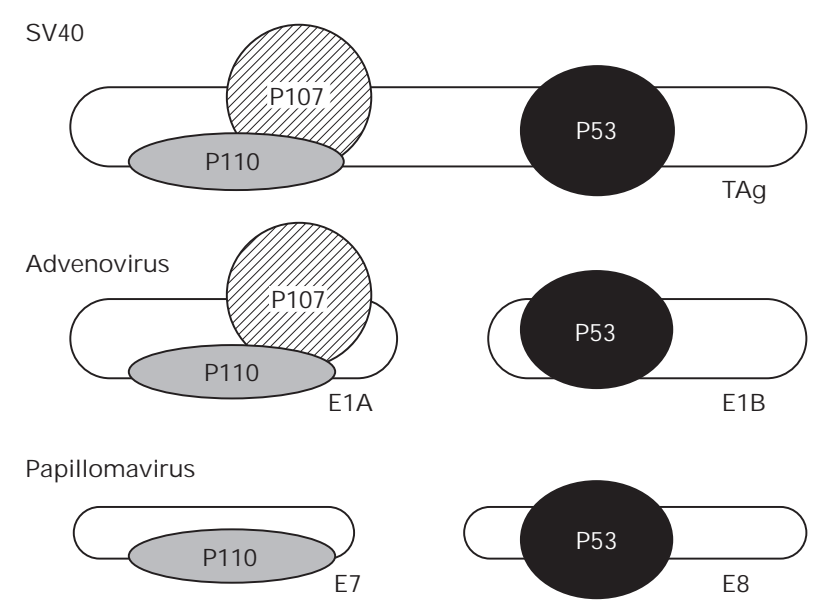

Figure 3 Representation of interactions between viral early antigens and host tumour suppressor gene products (p110, p53, plo7). Each virus has structurally similar elements two separate proteins are involved. Adapted with permission from Tannock and Hill. ${ }^{29}$

genes coding for growth factors, growth factor receptors, and intracellular signalling proteins are recognised and abnormalities of these genes are associated with a wide variety of malignancies. ${ }^{29} \mathrm{M}$ utations of proto-oncogenes to a more active state, duplication, or translocation to a position close to a pr stimulus to unregulated growth. This, in turn, provides greater opportunity for further gene abnormalities to develop. Inactivation of growth inhibitory proteins by parallel mechanisms or by binding to viral proteins can have the same range of effects. Alteration to a single gene is probably not sufficient to release full malignant expression. F or example, fibroblasts transfected with an abnormal myc gene become immortalised but remain phenotypically normal. If transfected with an abnormal ras gene they become phenotypically abnormal but retain a normal lifespan. Transfection with both leads to abnormal immortal cells which can cause tumours when injected into animals, though the tumours grow to a limited size only, suggesting that a further genetic abnormality is necessary for full malignant expression ${ }^{29}$

Disruption of the genome is an important feature of mesotheliomas with chromosomal abnormalities being identified in approximately $70 \%$ of tumours. ${ }^{32}$ The mechanism through which asbestos causes this damage mechanism through which asbestos causes this damage
is not known. Surface iron molecules can catalyse the generation of hydroxy radicals from intracellular hydrogen peroxide ${ }^{33}$ and other potentially toxic free radicals can be produced by phagocytosis-induced augmentation of the respiratory burst, but these chemaugmentation of the respiratory burst, but these chemof the physical properties of fibres in determining their of the physical properties of fibres in determining their
malignant potential. Asbestos can cause physical dismalignant potential. Asbestos can cause physical dis-
ruption of the cellular cytoskeleton and this may lead ruption of the cellular cytoskeleton and this may lead
to chromosomal instability during cell division, and might directly break or damage chromosomes leading to alterations to the genetic material. ${ }^{32}$ In vitro, cultured mesothelial cells ingest asbestos fibres, develop chromosomal abnormalities, and have a prolonged lifespan ${ }^{34}$ They do not, however, produce tumours when injected into animals. ${ }^{34}$ Although cells with chromosomal abnormalities are more easily transformed by SV $40,{ }^{35} \mathrm{CO}-$ exposure with SV 40 and asbestos is not sufficient to produce malignant cells using this model. ${ }^{27}$

Facilitation of entry of foreign DNA is another potential mechanism through which asbestos can lead to malignant change. F ree D N A can be detected in blood and bronchoalveolar lavage fluid, and is probably present locally in high concentrations at sites of inflammation. In vitro, asbestos fibres are at least as effective at incorporating viral D N A into cells as standard calcium phosphate coprecipitation methods ${ }^{36}$ It is therefore perhaps relevant that the timing of the infected thereforepertict polio virus epidemic coincided with the peak exposure of the working population to asbestos (fig 1) as the presence of fibres might have led to incorporation of SV 40 specifically into airway or mesothelial cells.

M ost of the chromosomal abnormalities induced by asbestos appear to be random and a considerable degree of heterogeneity is observed both between tumours and between different parts of the same tumour. ${ }^{32} 37$ Some are observed more frequently than might be expected by chance and might be of aetiological significance, but overall there is little evidence of mutations of known oncogenes in malignant mesotheliomas. Abnormalities of the retinoblastoma gene or the p53 gene do not appear to be important..$^{38} \mathrm{O}$ the other hand, immunohistochemical staining for $p 53$ has been reported in $35-70 \%$ of mesothe nce of abnormalities of the gene, and this p53 antigen appears to be of wild type rather than mutant. ${ }^{40}$ Pass and colleagues have recently reported a correlation between SV 40 large T antigen and p53 levels in mesotheliomas, offering further support for the hypothesis that SV 40 contributes to malignancy by binding and inactivating p53 and other nuclear proteins. ${ }^{39}$

\section{Conclusions}

Thereports of SV 40-like D N A in human mesotheliomas require confirmation, and the state and location of the viral genome need to be further characterised. H owever, the current information raises the possibility that SV 40 is an important cofactor in the development of mesotheliomas. If evidence of seropositivity or viral DNA is present in about $5 \%$ of the general population and in $60 \%$ of those with mesotheliomas, then that suggests an approximately 30 -fold increased risk of malignancy for those carrying the virus. Only limited reassurance can be taken from the cohort studies of populations exposed to SV40 as they all had limited power to detect even a large effect on a rare tumour. D emonstrating the the same as demonstrating an aetiological role but plausible molecular mechanisms exist which could explain an oncogenic role for SV 40 . The virus belongs to a class known to be potently tumorigenic in animals and has structural similarities to papilloma viruses which are associated with human cervical cancer. There is, however, no evidence that other papovaviruses or adenoof SV 40-like DNA in human tumours are confirmed, it would be an important step in viral oncology. A reliable serological test for SV 40 large T antigen would provide the most convenient means of investigating the observation epidemiologically.

If the observation is confirmed then immunohistochemical staining for TAg offers the potential for a useful diagnostic test. M ore importantly, it would provide a greater understanding of the molecular characteristics of a tumour which, to date, has proved resistant to all forms of treatment. Immunotherapy targeted at TAg might have a therapeutic role as might biochemical interference with the actions of TAg. It is known, for example, that the insulin growth factor 1 receptor is necessary for SV 40 cell transformation and 


\section{LEARNING POINTS}

* Malignant mesotheliomas currently cause approximately 1000 deaths per year in the UK and the numbers are predicted to rise until about the year 2020.

* Contaminated polio vaccines exposed a substantial proportion of the population to Simian virus 40 (SV40) between 1958 and 1961, and Simian virus DNA has been detected in subsequently arising human tumours.

* Simian virus 40 can induce malignant mesotheliomas in experimental animals.

* Simian virus 40 large T antigen (TAg) can bind and inactivate tumour suppression gene products and so allow cells to escape from normal grow th control.

* There is thus circumstantial evidence which suggests that Simian virus $\mathbf{4 0}$ may act as a cofactor with asbestos in inducing human mesotheliomas.

agents targeted at this molecule might have a useful therapeutic effect ${ }^{39}$ It would be paradoxical if a series of fortuitous findings which linked an unforeseen effect of polio vaccination to an unforseen effect of asbestos exposure was to provide the clue which led to an effective treatment for malignant mesotheliomas.

1 Peto J, Hodgson JT, M atthews FE, Jones JR. Continuing increase in 2 Hodgson IT Jones RD. M ortality of asbestos workers in England and Wales 1971-81. Br J ind M ed 1986;43:158-64.

3 Rudd R. Asbestos and lung cancer. Thorax 1997; 52:30

C, Jasani B, Navabi H, Wynford-T homas D, Gibbs AR Simian plification in human pleural mesothelioma tissue. Thorax 1996:51

5 Carson DA, Lois A. Cancer progression and p53. Lancet 1995;346

6 Bergsagel DJ, Finegold FJ, Butel JS, Kupsky WJ, Garcea RL. DNA sequences similar to

Lednicky JA, G arcea RL, Bergsagel DJ, Butel JS. N atural simian virus 40 strains are present in human choroid plexus and ependymoma tumors. Virology 1995;212:710-7.

8 Carbors tumours. Oncogene $1996 ; 13: 527-35$.
$M$ artini $F$, D e M attei $M$, laccheri $L$. Human brain tumours and simian virus 40. I N att Cancer Inst 1995;87:1331.

Tognon M, C asalone R, M artini F. Large T antigen coding sequences mosome changes in two glioblastoma cell lines. Cancer $G$ enet Cytogene1996;90:17-23. Carbone $M$, Pass $H$, Rizzlo $P$, M arinetti MR, mesothelioma. Oncogene 1994;9.1781-90.
Cristado A, Vivaldi A, Sensales G, Guglielmi G, Cianci E, Eliseri R, Cristado A, Vivaldi A, Sensales $G$, $G$ uglielmi $G$, Cianci 2 , Eliseri $R$,
et alecular biology studies on mesothelioma tumor samples preliminary data on $\mathrm{H}$-ras, P21, and SV40. J Exp Pathol Toxicol O nce $13 \mathrm{G}$ ardner $S$. Prevalence in England of antibody to human polyoma virus 14 Cicala C BMJ 1973;1.77-8.

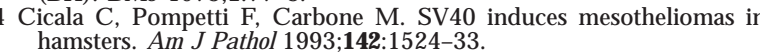
15 Strickler HD, Goedert J), F leming M. Simian virus 40 and pleural 5:473-5. $16 \mathrm{~K}$ rainer $\mathrm{M}$, Schenk T, Zielinski CC, M uller C. Failure to confirm
presence of SV 40 sequences in human tumours. Eur J Cancer 1995; presence of SV 40 sequences in human tumours. Eur J Cancer 1995;
31A:1893. 17 Sweet BH, Hilleman M R. T
Biol M ed 1960;105:420-7.

18 Shah K, N athanson N . H uman exposure to SV 40: review and comment

A $\mathrm{m}$ J Epidemiol 1976; 103:1-212.
19 Shah $\mathrm{K}$. Evidence for an SV 40 -related papova virus infection of man.
Am J E pidemiol 1972;95:199-206.
20 Eddy $B E$, Borman $G S$, Grubbs $G E$, Young RD. I dentification of the Virogegy 1962: $17: 65-75$.

$H$ einonen OP, Shapiro S, M onson R, H artz C, Rosenberg, Sloane D. Immunization during pregnancy against poliomyelitis and influenza 22 Pennisi $E$. M onkey virus DNA found in rare human cancers. Science

1997; 275: 748-9.
23 Soriano $F$, Shelburne, Gokcen M. Simian virus 40 in a human cancer.
$N$ ature 1964:249.421-5. $24 \mathrm{~K}$ Nirschstein RL, G erber P. Ependymomas produced after intracerebral
innoculation of SV40 into newborn hamsters. N ature 1962; 195:299300 25 Brinster RL, Chen HY, M essing A, van D yke T, Levine AJ, Palmiter acteristic brain tumors. Cell 1984;37:367-9. $26 \mathrm{KeY}$, Reddell IRR,$G$ erwin $B \mathrm{BI}$, Reddell $\mathrm{HK}$, Somers ANA, M CM enamin
$\mathrm{MG}$, et al. Establishment of a human in vitro mesothelial cell model $M G$, et al. Establishment of a human in vitro mesothelial cell model
system for investigating mechanisms of asbestos-induced mesosystem for investigating mechanisms of asbestos-induced meso-
thelioma. A m J Pathol 1989;134:979-91. Reddell RR, M alan-Shibley, $L$, Gerwin BI, M etcalf RA, $H$ arris $C C$.
T umorigenicity of human mesothelial celline transfected with EJ-ras oncogene. J $N$ atl Cancer Inst 1989,81945-8. 29 Tannock IF, Hill RP. The basic science of oncology. N ew York: M CG raw30 Shimizu E C Oxon A Otterson GA, Steinberg S, Kratzke RA, Kim YW, et al. RB protein status and clinical correlations from 171 cell lines
representing lung cancer, extrapulmonary small cell carcinoma, and

31 Selivanova G, Wiman K G. p53: a cell cycle regulator activated by D N A $32 \mathrm{Pass} H I$, M ew DJY. In vitro and in vivo studies of mesothelioma. J Cell

Fignard I, Pezerat H. Formation of asbestos. Can J Chem 1987:65:2338-41. 34 Lechner JF, Tokiwa T, LaVeck M, Banks-Schlegel S, Yeager H, Banarjee A, elal. A sbestos-associated chromosomal changes
thelial cells. Proc N atl A cad Sci U SA 1985;82:388-8

35 Torado AM, M artin G M. Increased susceptibility of D own's syndrome
fibroblasts to transformation by SV 40. Proc Soc Exp B iol M ed 1967; 124:1232-7. A ppell JD, Fasy T M, Kohtz D S, Johnson EM. A sbestos fibers mediate
transformation of monkey cells by exogenous plasmid D N A. P roc N at transtormation of monkey cells by exogenous plasmid DNA. Proc Nat
A cad Sci S 1988:85:7670-4 37 A uncharek M G Genetic factors in

thelioma. Eur J Cancer 1995;31A:1741-7. $M$ or $O$, Yaron $P$, Huszar $M$, Yellin A, Jakobovitz $O$, Brok-Simoni $F$, et
al. Absence of p53 mutations in malignant mesotheliomas. Am J Respir C Cll M ed Biol 1997:16:9-13. Pass HI, Kennedy RC, C Carbone M. Evidence for and implication of SV 40 -like sequences in human mesotheliomas. In: D eVita VT, Hell-
man S, Rosenberg SA, eds. I mportant advances in oncology. PhilaTooze J, ed. M olecular biology of tumor viruses: D N A tumor viruses. 2nd
edn. Cold Spring H arbor Laboratory Press, 1981 . 